\title{
Gut microbiota and probiotics in modulation of epithelium and gut-associated lymphoid tissue function
}

Microbial Ecophysiology and Nutrition Group. Institute of Agrochemistry and Food Technology (IATA). Spanish National Research Council (CSIC). Valencia, Spain Yolanda Sanz* and Giada De Palma

Running head: Gut microbiota and mucosal immunity

*Corresponding author: Yolanda Sanz

Microbial Ecophysiology and Nutrition Group, Institute of Agrochemistry and Food Technology (IATA). Spanish National Research Council (CSIC). PO Box 73, 46100 Burjassot-Valencia, Spain

Tel: 34963900022

Fax: 34963636301

E-mail:yolsanz@,iata.csic.es 


\begin{abstract}
The intestinal tract mucosa is exposed to a vast number of environmental antigens and a large community of commensal bacteria. The mucosal immune system has to provide both protection against pathogens and tolerance to harmless bacteria. Immune homeostasis depends on the interaction of indigenous commensal and transient bacteria (probiotics) with various components of the epithelium and the gut-associated lymphoid tissue (GALT). Herein, an update is given of the mechanisms by which the gut microbiota and probiotics are translocated through the epithelium, sensed via pattern-recognition receptors, and activate innate and adaptive immune responses.
\end{abstract}

Key-words: gut microbiota, probiotics, mucosal immunity, regulatory $\mathrm{T}$ cells. 


\section{Introduction}

The intestinal tract mucosa is exposed to a vast number of environmental antigens acquired orally and a large community of commensal bacteria. Herein, the epithelium, the mucosal immune system and the gut microbiota orchestrate a network of immunological and nonimmunological defences, providing both protection against pathogens and tolerance to commensal bacteria and harmless antigens. The intestinal epithelium constitutes a physical barrier that excludes most of the antigens prior to immune activation. The primary defensive mechanisms also include the production of mucus and other secretions, regulation of paracellular permeability and synthesis of antimicrobial peptides. In addition, the epithelium and the gut-associated lymphoid tissue (GALT) develop complex immune responses to different stimuli, which involve the production of cytokines, chemokines and different effector and regulatory $\mathrm{T}$ cells, which ensure pathogen elimination without causing tissue damage in physiological conditions.

The gut microbiota and transient bacteria (probiotics) are known to influence the development and regulation of the host's defences, of immune and non-immune nature. Commensal bacteria regulate mucin gene expression (e.g. MUC-2 and MUC-3 genes) by goblet cells and modify the glycosylation pattern, which may affect bacterial adhesion, colonization and invasion $[1,2]$. Some commensal bacteria also induce the secretion of antimicrobial peptides (defensins and angiogenins) by intestinal Paneth cells [3, 4] and regulate the alterations of permeability associated with infections, stress and inflammatory conditions [5]. Moreover, comparisons between germ-free and colonized mice showed that microbiota has an enormous impact on the development of mucosal and systemic immunity In germ-free mice, the GALT was immature, with a low content of lamina propria $\mathrm{T}$ cells, immunoglobulin (Ig) A producing $\mathrm{B}$ cells and intraepithelial $\mathrm{T}$ cells. Absence of the microbiota also affected systemic immunity since germ-free mice had decreased serum 
immunoglobulin levels and smaller spleens [6]. These differences disappeared after colonization with the commensal microbiota or administration of bacterial components, which are ligands of innate immune cell receptors [7]. Some probiotic bacteria can also stimulate the immune system enough to enhance defences against acute infections and improve certain chronic inflammatory bowel conditions in humans [8-11]. Herein, the mechanism by which the gut microbiota and probiotics are translocated and interact with components of the epithelium and the GALT, thereby regulating the host's innate and adaptive immune responses, are reviewed.

\section{Intestinal epithelium and mucosal immune system}

The intestinal epithelium is a selective barrier that separates the luminal content from the immune cells of the underlying lamina propria. It consists of a single layer of epithelial cells, which include enterocytes (representing 90-95\%), mucus-secreting goblet cells, enteroendocrine cells and Paneth cells secreting antimicrobial peptides and proteins. The intestinal epithelial cells (IECs) develop a barrier function of non-immunologic nature that involves secretion of mucus and antimicrobial peptides (e.g. defensins and angiogenins) and regulation of cell turnover and integrity. Absorptive cells are involved in the transport and internalization of substances (transcytosis) by non specific and specific receptor-mediated mechanisms. In this way, IECs import luminal nutrients and antigens, and release IgA into the lumen [12]. IECs are also sealed by tight junctions, which regulate the paracellular transport of specific substances. In addition, the IECs also develop immune functions and express co-stimulatory molecules and components of the human major histocompatibility complex, (MHC) and produce inflammatory mediators in response to a stimulus (e.g. TNF, IL8, MCP-1, etc.). IECs participate in the initiation and regulation of the mucosal immune 
response via interactions with professional immune cells found in the epithelium and lamina propria, a layer of connective tissue supporting the epithelium [13].

The GALT consists of variety of cells (lymphocytes, dendritic cells [DCs], macrophages, etc.), often grouped in organised structures such as Peyer's Patches (PPs), mesenteric lymphoid nodes (MLN), and isolated lymphoid follicles [14]. Intestinal PPs are more permeable to bacteria than other epithelial areas since they lack an overlying brush border, mucoid glycocalyx and hydrolytic enzymes, which are characteristic of absorptive epithelium, and have specialised transport cells, such as M cells and DCs [15]. Immune cells include B lymphocytes (memory cells and plasma cells), of which $70-90 \%$ produce type A immunoglobulins, and T lymphocytes, which are classified as CD4+ (helper or inducer) and CD8+ (suppressor or cytotoxic) cells. CD4+ lymphocytes are mainly in the lamina propria while CD8+ lymphocytes are mainly in the epithelia (intraepithelial T cells).

The first portal of entry of antigens is through enterocytes, M cells and DCs. Epithelial cells and antigen presenting cells (DCs and macrophages) express pattern-recognition receptors, which are responsible for the initial recognition of specific microbial components (pathogenassociated molecular patterns) and the discrimination between pathogens and harmless microbes and the development of appropriate innate and acquired immune responses [16]. These receptors include the Toll-like receptor family (TLRs), which are located at the cell surface (e.g. TLR 1, 2, 4 and 5) and intracellularly (e.g. TLR3, 7, 8 and 9), and Nod-like receptors (NLRs), which are located intracellularly [17, 18]. For example, TLR2 recognizes lipoteichoic acids (LTA) from Gram-positive bacteria, TLR4 recognizes lipopolysaccharide (LPS) from Gram-negative bacteria, TLR9 recognizes special sequences of DNA (unmethylated $\mathrm{CpG}$ motifs), TLR5 recognizes flagellin and NLRs appear to recognize bacterial peptidoglycans (Fig. 1). TLRs are transmembrane proteins that upon-ligand binding promote signal divergence via interactions with different adaptor proteins (MyD88, 
MAL/TIRAP, TRIF/TICAM-1, TRAM/TIRP/TICAM-2), thereby activating three major signalling pathways: nuclear factor (NF)- $\kappa \mathrm{B}$, the mitogen-activated protein kinases (MAPKs) and interferon regulatory factors (IRFs) (Fig 1.) [19]. This leads to the expression of inflammatory genes, including those encoding cytokines, cytokines receptors, immunoregulatory proteins, adhesion molecules, stress-associated proteins and other mediators. Overall these molecules are involved in the recruitment of other immune cells (T cells, basophils, neutrophils, dendritic cells and natural killer cells) and in promoting inflammatory responses that can lead to pathogen clearance. NOD1 and NOD2 are the bestcharacterized NLRs and play a role in detecting of intracellular microorganisms. NOD1 is expressed in all cell types and required for NF- $\kappa \mathrm{B}$ activation by Gram-negative bacterial infection, once the bacteria have bypassed TLR activation [20]. NOD2 is expressed in monocytes/macrophages and DCs and is induced in intestinal epithelial cells by TNF- $\alpha$. NOD2 mutations are associated with defective IL-10 production and Crohn's disease [21].

Signalling through TLR also stimulates the maturation of DCs, inducing their antigen presentation ability, switching on the chemokine receptor program and allowing cells to migrate into draining MLN, where they present antigens to naïve $\mathrm{T}$ and $\mathrm{B}$ cells. T-cell differentiation into Th1, Th2 or regulatory $\mathrm{T}$ cells $(\mathrm{Th} 3 / \mathrm{Tr} 1)$ is also thought to depend on the type of TLRs involved and cytokine production (Fig. 2). Th1-biased responses, characterized by overproduction of IFN- $\gamma$, IL-2, and IL-12 cytokines, are associated with inflammatory reactions and clearance of intracellular pathogens as well as with chronic inflammatory bowel disease such as Crohn's disease. Most TLR-activated DCs induce differentiation of naïve CD4+ T cells into Th1 cells, providing high levels of the Th1-polarizing cytokine IL-12, whereas TLR5 and TLR2-activated DCs may promote the differentiation of Th2-cells or regulatory $\mathrm{T}$ cells by producing high levels of anti-inflammatory cytokine IL-10 and low levels of IL-2 [19, 22]. Antigen-specific regulatory T cells include different subtypes of 
CD4+ T cell: T regulatory $1(\operatorname{Tr} 1)$ cells, which secrete high levels of IL-10, no IL-4, and no or low levels of IFN- $\gamma$; and T helper 3 (Th3) cells, which secrete high levels of TGF- $\beta$ [23]. In the absence of inflammation, a balance between effector and regulatory lymphocyte subpopulations is maintained through a tightly controlled cytokine network. Both IL-10 and TGF- $\beta$ are important cytokines in directing naïve $\mathrm{T}$ cell maturation to the generation of regulatory $\mathrm{T}$ cells.

\section{Translocation of commensal and probiotic bacteria}

Translocation of certain numbers of indigenous bacteria to the MLN seems to be a physiological process, which allows the interaction of luminal bacteria with immune competent cells, underlying the epithelium, and the induction of different immune responses. In pathogen-free mice challenged with intestinal doses of commensal bacteria, small numbers of commensals were shown to bind to the luminal side of the $\mathrm{M}$ cells, penetrate the epithelial cell layer and survive within DCs of intestinal MLN [24]. After capture by DCs, the activation of $\operatorname{IgA}$ responses was triggered locally and at distant mucosal sites, which may contribute to the role of commensal and probiotic bacteria in the neutralization of noxious antigens and pathogens as well as in the development of tolerance to harmless bacteria [25]. Enterobacter cloacae, used as a model of commensal bacteria and administrated to mice, was shown to be sampled by M cells in PP and then phagocytosed by CD11c+ DC, which were activated and expressed different co-stimulatory molecules such as CD86 [25]. The probiotic E. coli Nissle 1917 was also shown to be translocated into PP and MLN after oral administration to mice [26]. Lactobacillus casei strain Shirota was incorporated to M cells of PP and digested by innate immune cells to form bioactive components [27]. These bioactive components were then recognized through TLR2 in antigen-presenting cells, which thus became able to produce different cytokines and, especially, TNF- $\alpha$. Moreover, the expression 
level and/or the phosphorylation of some proteins in PPs and MLN were modified by the ingestion of the probiotic. In weaned piglets fed with a strain of Bifidobacterium animalis, DNA from Bifidobacterium was also detected in MLN and its concentration increased as the probiotic dose increased [28]. L. plantarum Lp6 intake was also able to modify the gene expression of jejunal Peyer's patches, including genes involved in immune response, cell differentiation, cell-cell signalling, cell adhesion, and signal transcription and transduction [15].

\section{TLR expression and signalling}

TLR expression, localization and signalling in IECs and immune cells are critical to mucosal immune activation in the gut, and constitute key elements regulated by the indigenous microbiota and probiotic bacteria (Fig 1).

In recent years, several studies have reported that Gram-negative and Gram-positive bacteria, as well as commensal and pathogenic bacteria, differently influence TLR expression and, thereby, their mediated immune responses. In mice, Gram-positive bacteria (L. acidophilus CRL 924, L. delbrueckii subsp. bulgaricus CRL 423 and the probiotic L. casei CRL 431) increased the number of TLR2 positive cells, while Gram-negative bacteria (E. coli 129 and E. coli 13-7) increased that of TLR4 positive cells parallel to different cytokine induction (IL10 versus IL-12) [29]. L. plantarum BFE 1685, isolated from a child's faeces, and the probiotic strain L. rhamnosus GG, but not S. enterica serovar Typhimurium, up-regulated TLR2 and TLR9 transcription levels in HT29 cells [30]. Moreover, protein levels of TLR2 and TLR5 were enhanced by the lactobacilli strains [30]. The Gram-negative probiotic strain E. coli Nissle 1917 in co-culture with human T cells increased TLR2 and TLR4 protein expression and NF- $\mathrm{KB}$ activity. In wild-type mice, but not in TLR2 or TLR4 knockout mice, this probiotic ameliorated colitis and decreased pro-inflammatory cytokine secretion, 
suggesting that the effects were mediated via TLR2- and TLR4-dependent pathways [31]. However, Gram-positive probiotic bacteria mainly induce TLR2 expression via interactions with LTAs [32], but not TLR4 expression. For example, the administration of an aggregating strain of L. crispatus M247 to mice increased TLR2 mRNA levels and reduced TLR4 mRNA and protein levels in the colonic mucosa through an extracellular signal-regulated kinase-1 (ERK1) tyrosine phosphorylation-dependent pathway [33]. In colonic epithelial cells, preexposure to L. crispatus M247 also inhibited the LPS-induced IL-6 release and enhanced TLR2-mediated IL-10 up-regulation. When mice were administered a strain of B. animalis together with fructo-oligosaccharides the dose of $B$. animalis linearly influenced TLR2 gene expression in the lymph nodes and the bifidobacterial DNA negatively correlated with the TNF- $\alpha$ gene expression [28]. Despite sharing certain features, Gram-positive pathogenic and commensal bacteria influence TLR expression differently. Both L. rhamnosus GG and the human pathogen Streptococcus pyogenes enhanced TLR2 expression in macrophages and required TLR2 for NF-אB activation. However, only the pathogenic S. pyogenes up-regulated TLR3 and TLR7 gene expression [34].

DNA of probiotic bacteria also modulates TLR9 expression and thereby elicits a differential response in epithelial and immune cells compared with DNA of pathogenic bacteria in vitro and in vivo $[35,36]$. Exposure of HT-29 cells to DNA of pathogenic strains of Salmonella and E. coli led to a significant increase in TLR9 mRNA expression and Salmonella enterica serovar Dublin DNA also increased surface TLR9 protein and IL-8 secretion, whereas exposure to Bifidobacterium breve DNA did not elicit any change in mRNA levels or TLR9 localization [36]. The DNA from some probiotic bacteria also limited epithelial proinflammatory responses in vivo and in vitro via TLR9 modulation. In HT-29 cells subjected to pro-inflammatory stimuli, DNA from the probiotic mixture VSL3 inhibited IL-8 secretion, reduced p38 MAPK expression, delayed NFкB activation, stabilized levels of $\mathrm{I} \kappa \mathrm{B}$, and 
inhibited proteasome function [35]. In wild-type mice, VSL3 DNA attenuated a systemic release of TNF- $\alpha$ in response to E. coli DNA injection and, in IL-10-deficient mice, oral VSL3 DNA administration led to a reduction in mucosal secretion of TNF- $\alpha$ and IFN- $\gamma$, improving the histological disease via TLR9 signalling [35].

Commensal bacteria and some probiotics also influence the expression of negative regulators of TLR-mediated immune activation, such as the Toll-interacting protein Tollip, the peroxisome-proliferator-activated receptor (PPAR) $\gamma$ and the A20 protein. Prolonged incubation of IECs with TLR ligands (LPS and LTA), resulted in a state of hyporesponsiveness associated with increased Tollip mRNA. This would suggest that exposure to commensal bacteria could regulate the expression of this negative regulator, which inhibits signalling via TLR2 and TLR4 and suppresses LPS-induced IL-1 receptor associated kinase (IRAK) phosphorylation and activity, impairing transcriptional activity of $\mathrm{NF} \kappa \mathrm{B}$ and AP-1. AP-1. This mechanism could therefore contribute to intestinal homeostasis [37]. PPAR- $\gamma$ is a member of the steroid-receptor family, which plays a major role in maintaining intestinal mucosa homeostasis and whose expression and function are modulated by TLR signalling and the gut microbiota. $B$. thetaiotaomicron induces PPAR- $\gamma$ expression and triggers PPAR- $\gamma$ mediated nuclear export of the transcriptionally active RelA subunit of the NF- $\kappa \mathrm{B}$, attenuating the inflammatory effects induced by Salmonella enteritidis in IECs [38]. L. crispatus M247 supplementation in mice was also shown to increase PPAR- $\gamma$ levels and reduce activity of an NFkB-responsive element in the colonic mucosa [39]. This strain uses $\mathrm{H}_{2} \mathrm{O}_{2}$ as a signal transducing molecule to induce PPAR- $\gamma$ activation in IECs. A20 protein is another negative regulator of NFKB and TLR signalling that is inducible by cytokines. The administration of $B$. lactis $\mathrm{Bb} 12$, but not of $B$. vulgatus, to rats stimulated A20 mRNA expression in primary and IECs lines.

[40]. 
The gut microbiota also regulates the activation of different components of the TLR signalling pathways. Commensal bacteria and some probiotics attenuate the proinflammatory responses by transient activation or inhibition of the NF- $\kappa \mathrm{B}$ signalling pathway at different steps. B. thetaiotaomicron acts downstream NFkB activation by promoting

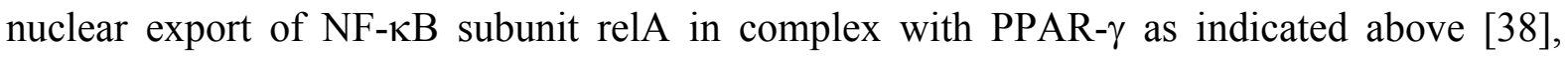
while other commensal bacteria block NFkB at more proximal steps, inhibiting ubiquitination

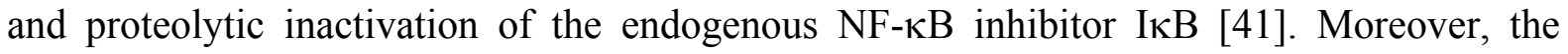
probiotic strain L. reuteri ATCC PTA 6475 can suppress TNF production by LPS-activated monocytes and primary monocyte-derived macrophages from children with Crohn's disease by inhibiting the activation of MAP kinase-regulated c-Jun and the transcription factor AP-1 [42]. Exposure of IECs to bacterial LPS has also been shown to induce TLR tolerance in these cells via posttranscriptional down-regulation of the interleukin 1 receptor-associated kinase 1 (IRAK1), which proved essential for epithelial TLR4 signalling in vitro [43]. Bacterial cells and soluble factors released by Bifidobacterium breve $\mathrm{C} 50(\mathrm{Bb})$ induced a dose-dependent inhibition of the chemokine CXCL8 secretion by epithelial cells (HT-29) driven by both $\mathrm{AP}-1$ and $\mathrm{NF}-\kappa \mathrm{B}$ transcription pathways, which implies decreased phosphorylation of p38-MAPK and IкB- $\alpha$ molecules. In trinitro-benzene sulphonic acid (TNBS)-induced colitis in mice, soluble factors of this strain decreased the colitis score and inflammatory cytokine expression, by inhibiting phosphorylations involved in inflammatory processes and by exerting protective effects on DCs [44]. Bacterial signalling to NOD receptors has also been shown to inhibit the TLR2-driven activation of NF-kB signalling and its deficient functioning is associated with Crohn's disease [45].

\section{Cytokine induction}


Commensal, probiotic and pathogenic bacteria modulate cytokine production by epithelial and professional immune cells differently, contributing to the protective immune response against pathogen invasion and the regulation of TLR expression. Several studies indicate that some probiotics and commensal bacteria can stimulate protective immune responses to enhance resistance to microbial pathogens, which involved the induction of pro-inflammatory cytokines and chemokines production (e.g. TNF- $\alpha$, IL-8, IL-12 and IFN- $\gamma$ ) [46]. For example, the probiotic strain L. casei Shirota has been shown to induce IL-12 and IFN $\gamma$ expression in mouse splenocites, which could help fight against pathogens [47]. L. plantarum BFE 1685 and the probiotic strain L. rhamnosus GG also lead to increased IL-8 production in response to S. Typhimurium in HT29 cells, indicating that these cells are sensitised by lactobacilli to enhance their defence against the pathogen [30]. L. reuteri 100-23 administered to mice induced a transient gene expression of pro-inflammatory cytokines and chemokines, including IL-1 $\alpha$, IL-6, IFN- $\gamma$ inducible protein 10 , and macrophage inflammatory protein 2 [48]. Modulation of cytokine production by commensal bacteria and pathogens also influences TLR expression. For example, IFN- $\gamma$ was found to increase mRNA and surface expression of TLR4 in human monocytes and macrophages, enhancing their responsiveness to LPS in terms of phosphorylation of IRAK (immediately downstream of the MyD88 adapter protein), NF-kB DNA binding activity, and cytokine (TNF- $\alpha$ and IL-12) production [49]. This enhanced TLR4 expression probably contributes to pathogen recognition and killing by mononuclear phagocytes.

Commensal and probiotic strains could also regulate the degree of immune activation in response to pathogens or other harmful antigens, preventing excessive inflammation. These regulatory effects may be mediated by the induction of regulatory and anti-inflammatory cytokine production (IL-10 and TGF- $\beta$ ), which trigger suppressive pathways via modulation of TLR-signalling and lymphocyte differentiation (described below). Several commensal and 
probiotic strains have been shown to induce IL-10 in epithelial and immune cells in vitro and in vivo $[50,51]$. In the epithelium, IL-10 triggers different anti-inflammatory mechanisms through JAK1/STAT3 and p38 MAPK-dependent pathways, and may also directly confer protection by regulating the endoplasmic reticulum stress response linked to the activation of $\mathrm{NK} \kappa \mathrm{B}$ pathway and production of reactive oxygen species, involved in chronic inflammatory pathologies [52]. TGF- $\beta$ is produced at an early state of bacterial colonization in animal models [40] and induced by the intake of some probiotic strains (e.g. B. longum 2C and 46 and B. lactis $\mathrm{Bb}-12$ ) in human peripheral blood [53]. TGF- $\beta$ may regulate inflammatory responses by inhibiting the TLR-induced NF-kB-dependent pro-inflammatory gene expression program through the induction of TLR2 degradation via Smad signalling [52]. It has been hypothesised that commensal bacteria produce a transient activation of $\mathrm{NK \kappa \textrm {B }}$ pathway in epithelial cells that triggers IL-10 and TGF- $\beta$ mediated responses in the epithelium and lamina propria, thus contributing to maintaining immune homeostasis to commensal bacteria in the gut. Moreover, studies in germ-free and pathogen-free mice have shown that microbial colonization induced the expression of antimicrobial peptides but down-regulated the expression of pro-inflammatory type I IFN related genes in the large intestine, which could be an additional mechanism to prevent excessive inflammation due to continuous microbial exposure [54].

\section{Lymphocyte differentiation and regulatory $T$-cell generation}

Lymphocyte differentiation may also be influenced by certain probiotic strains and commensal bacteria contributing to the host's defences against pathogen infection, but avoiding excessive immunostimulation, which would lead to tissue damage [55]. TLRs and co-stimulatory molecules expressed by DCs and the cytokine network are essential to the induction of a balanced differentiation of naïve $\mathrm{T}$ cells into effector $\mathrm{T}$ cells (Th1 and $\mathrm{Th} 2)$ 
and IgA-secreting B cells, required to fight off pathogens, as well as into regulatory $\mathrm{T}$ cells (Tr1 and Th3) required to control excessive inflammation (Fig. 2) [56].

Probiotic and commensal bacteria induce different cytokine production by stimulation of DCs in comparison with pathogenic bacteria, which may explain their different effects on lymphocyte differentiation. For example, O'Mahony et al. [57] reported that commensal bacteria (Lactobacillus and Bifidobacterium) induced regulatory cytokine production (IL-10) by MLN and MLN-derived DCs cells, whereas pathogenic bacteria (Salmonella) induced Th1-polarizing cytokines (IL-12 and TNF- $\alpha$ ). IL-10 production and CD83 expression were also induced by strains of the species B. bifidum, B. longum, and B. pseudocatenulatum in cord blood DCs, polarising the immune response toward a Th-2 profile [58]. B. breve $\mathrm{BbC} 0_{\mathrm{SN}}$ supernatant also induced DC maturation by up-regulating the expression of CD83, as well as CD86 and HLA DR, and IL-10 production, which involved TLR2 interactions [59]. Probiotic strains of the product VSL\#3, which include lactobacilli, streptococci and bifidobacteria, were also shown to influence DCs maturation. Individual strains displayed distinct immunomodulatory effects on DCs and the most marked anti-inflammatory effects were exerted by bifidobacteria, which up-regulated IL-10 production by DCs, decreased expression of the costimulatory molecules CD80 and CD40, and decreased IFN- $\gamma$ production by $\mathrm{T}$ cells [60]. In addition, different strains of the same species may polarize immune responses in different directions via cytokine production regulation $[50,51]$. While some $B$. longum strains induced a Th2 orientation with high levels of IL-4 and IL-10, both secreted by splenocytes, and of TGF- $\beta$ gene expression in the ileum of germ-free mice inoculated with the bifidobacteria, other strains induced Th1 orientations with high levels of IFN- $\gamma$ and TNF$\alpha$ splenocyte secretions [51].

The induction of regulatory $\mathrm{T}$ cells by commensal and some probiotic bacteria may contribute to suppressing activation of the immune system and, thereby, to maintaining 
immune homeostasis and tolerance to self and harmless exogenous antigens [61]. For example, strains of $L$. reuteri and $L$. casei, but not of $L$. plantarum were shown to prime human monocyte-derived DCs to trigger regulatory T-cell development [62]. These regulatory $\mathrm{T}$ cells produced increased levels of IL-10 and were able to inhibit the proliferation of bystander T cells in an IL-10-dependent fashion. The binding of both $L$ reuteri and $L$. casei the C-type lectin, which is a DC-specific intercellular adhesion molecule 3-grabbing non-integrin (DC-SIGN), was necessary to induce regulatory $\mathrm{T}$ cells [62]. $B$. infantis 35624 also induced regulatory $\mathrm{T}$ cells in mice, contributing to protecting the host against aberrant activation of the innate immune system in response to infection with Salmonella enterica serovar Typhimurium or injection with LPS [63]. In B. infantis-fed mice, a profound inhibition of infection and LPS-induced NF- $\mathrm{B}$ activity, which preceded a reduction in S. enterica serovar Typhimurium numbers, and murine sickness behaviour scores, was detected. In addition, pro-inflammatory cytokine secretion, T-cell proliferation, and DC co-stimulatory molecule expression were significantly reduced. In contrast, CD4+CD25+Foxp3 $+\mathrm{T}$ cell numbers increased significantly in the mucosa and spleen of mice fed $B$. infantis. In addition, adoptive transfer of $\mathrm{CD} 4+\mathrm{CD} 25+\mathrm{T}$ cells transferred the NFאB inhibitory activity. Transfer of probiotic-treated DCs to mice also protected against 2, 4, 6-trinitrobenzenesulfonic acid-induced colitis, partially, via induction of CD4+ CD25+ regulatory cells [64]. The preventive effect of probiotic-pulsed DCs required MyD88, pattern recognition receptors (TLR2- and NOD2) -dependent signalling and the induction of CD4+ CD25+ regulatory cells in an IL-10-independent fashion. These effects were induced by $L$. salivarius Ls33 and L. rhamnosus Lr32 but not by L. acidophilus NCFM. In contrast, the capsular polysaccharide of the Gram-negative bacteria Bacteroides fragilis proved to be taken up by CD11c+ DC, leading to the production of IL-12 and increased Th1 response [65]. 
Some probiotics and commensal bacteria may also activate local antigen presenting cells to enhance antigen presentation to B lymphocytes and increase secretory IgA production both locally and systemically, improving the host's defences against pathogens [66]. However, in pathogen-free mice commensal-loaded DC alone did not induce systemic immune responses because the commensal-loaded DCs were restricted to the mucosal immune compartment by the MLN [25]. Thus, pathogen-free mice remained systemically ignorant of their commensal microbiota. In fact, induction of secretory IgA against commensal bacteria is induced by both T-independent and T-dependent pathways, while induction of IgA against noxious stimuli (e.g. cholera toxin) is highly T-help dependent, reflecting the involvement of different immune responses to harmless and harmful microbial stimuli.

\section{Concluding remarks}

Scientific evidence supports a role for the microbiota and some probiotics in mucosal immunity regulation by taking part in different key events of the immune response, from TLR signalling to lymphocyte differentiation into regulatory $\mathrm{T}$ cells. Altogether, these contribute to improving the host's defences against harmful agents and to maintaining intestinal homeostasis. Accordingly, specific probiotic strains are acknowledged for their ability to increase the defences against acute infections, improve chronic inflammatory bowel conditions and induce tolerance to harmless environmental antigens. Nevertheless, the impact of probiotics on diverse disease conditions are more subtle than expected. Advances in the understanding of the mechanisms by which specific components of the gut microbiota induce tolerance or predispose to disease via interactions with specific molecules of the epithelium and the GALT would be of great help to identify the molecular targets of probiotics and the biomarkers of their effects, and to provide sounder evidences on their benefits on physiologic conditions and immune-mediated disorders. 


\section{Declaration of interest}

The authors report no conflicts of interest.

\section{Acknowledgements}

This work was supported by grants AGL2008-01440/ALI and Consolider Fun- $C$-Food CSD2007-00063 from the Spanish Ministry of Science and Innovation. The scholarship to G. De Palma from JAE-CSIC (Spain) is fully acknowledged. 


\section{References}

1. M. Freitas, L.G. Axelsson, C. Cayuela, T. Midtvedt and G. Truenan, Indigenous microbes and their soluble factors differentially modulate intestinal glycosylation steps in vivo. Use of a "lectin assay" to survey in vivo glycosylation changes. Histochem. Cell Biol., 124:423-33, 2005.

2. C. Caballero-Franco, K. Keller, C. De Simona and K. Chadee, The VSL\#3 probiotic formula induces mucin gene expression and secretion in colonic epithelial cells. Am. J. Physiol. Gastrointest. Liver Physiol., 292:G315-22, 2207.

3. L.V. Hooper, T.S. Stappenbeck, C.V. Hong and J.L. Gordon, Angiogenins: a new class of microbicidal proteins involved in innate immunity. Nat. Immunol., 4:269-73, 2003.

4. S. Vaishnava, C.L. Behrendt, A.S. Ismail, L. Eckmann and L.V. Hooper, Paneth cells directly sense gut commensals and maintain homeostasis at the intestinal host-microbial interface. Proc. Natl. Acad. Sci. USA., 105:20858-63, 2008.

5. F. Lutgendorff, L.M. Akkermans and J.D. Söderholm, The role of microbiota and probiotics in stress-induced gastro-intestinal damage. Curr. Mol. Med., 8:282-98, 2008.

6. H. Tlaskalová-Hogenová, R. Stepánková, T. Hudcovic, L. Tucková, B. Cukrowska, R. Lodinová-Zádníková, H. Kozáková, P. Rossmann, J. Bártová, D. Sokol, D.P. Funda, D. Borovská, Z. Reháková, J. Sinkora, J. Hofman, P. Drastich and A. Kokesová, Comensal bacteria (normal microflora), mucosal immunity and chronic inflammatory and autoimmune diseases. Immunol. Lett., 93:97-108, 2004.

7. S. Rakoff-Nahoum, J. Paulino, F. Eslami-Varzaneh, S. Edberg, R. Medzhitov, Recognition of commensal microflora by toll-like receptors is required for intestinal homeostasis. Cell., 118:229-41, 2004. 
8. M.C. Lee, L.H. Lin, K.L. Hung and H.Y. Wu, Oral bacterial therapy promotes recovery from acute diarrhea in children. Acta Paediatr. Taiwán., 42:301-5, 2001.

9. P. Gionchetti, F. Rizzello, U. Helwig, A. Ventura, K.M. Lammers, P. Brigidi, B. Vitali, G. Poggioli, M. Miglioli and M. Campieri, Prophylaxis of pouchitis onset with probiotic therapy: a double-blind, placebo-controlled trial. Gastroenterology, 124:1202-9, 2003.

10. S.J. Allen, B. Okoko, E. Martinez, G. Gregorio and L.F. Dans, Probiotics for treating infectious diarrhoea. Cochrane Database Syst. Rev., 2:CD003048, 2004.

11. W. Kruis, P. Fric, J. Pokrotnieks, M. Lukás, B. Fixa, M. Kascák, M.A. Kamm, J. Weismueller, C. Beglinger, M. Stolte, C. Wolff and J. Schulze, Maintaining remission of ulcerative colitis with the probiotic Escherichia coli Nissle 1917 is as effective as with standard mesalazine. Gut, 53:1617-23, 2004.

12. M. Heyman and S. Menard, Pathways of gliadin transport in celiac disease. Ann. NY Acad. Sci., 1165:274-8, 2009.

13. A. Izcue, J.L. Coombes and F. Powrie, Regulatory lymphocytes and intestinal inflammation. Annu. Rev. Immunol., 27:313-38, 2009.

14. J.P. Perón, A.P. Ligeiro de Oliveira and L.V. Rizzo, It takes guts for tolerance: The phenomenon of oral tolerance and the regulation of autoimmune response. Autoimmun. Rev., Feb 27. [Epub ahead of print], 2009.

15. G. Chang, Y. Shi, G. Le, Z. Xu, J. Sun and J. Li, Effects of Lactobacillus plantarum on genes expression pattern in mice jejunal Peyer's patches. Cell Immunol. 258:1-8, 2009.

16. K.S. Michelsen and M. Arditi, Toll-like receptors and innate immunity in gut homeostasis and pathology. Curr. Opin. Hematol., 14:48-54, 2007. 
17. L.H. Travassos, S.E. Girardin, D.J. Philpott, D. Blanot, M.A. Nahori, C. Werts and I.G. Boneca, Toll-like receptor 2-dependent bacterial sensing does not occur via peptidoglycan recognition. $E M B$,. 5: 1000-6, 2004.

18. L.C. Parker, L.R. Prince and I. Sabroe, Translational mini-review series on Toll-like receptors: networks regulated by Toll-like receptors mediate innate and adaptive immunity. Clin. Exp. Immunol., 147: 199-207, 2007.

19. M.S. Lee and Y.J Kim, Signaling pathways downstream of pattern-recognition receptors and their cross talk. Annu. Rev. Biochem., 76:447-80, 2007.

20. A. Fukazawa, C. Alonso, K. Kurachi, S. Gupta, C.F. Lesser, B.A. McCormick and H.C. Reinecker, GEF-H1 mediated control of NOD1 dependent NF-kappaB activation by Shigella effectors. PLoS Pathog. 4(11):e1000228, 2008.

21. M.G. Netea, B.J. Kullberg, D.J. de Jong, B. Franke, T. Sprong, T.H. Naber, J.P. Drenth and J.W. Van der Meer, NOD2 mediates anti-inflammatory signals induced by TLR2 ligands: implications for Crohn's disease. Eur. J. Immunol., 34:2052-9, 2004.

22. A. Didierlaurent, I. Ferrero, L.A. Otten, B. Dubois, M. Reinhardt, H. Carlsen, R. Blomhoff, S. Akira, J.P Kraehenbuhl and J.C. Sirard, Flagellin promotes myeloid differentiation factor 88-dependent development of Th2-type response. J. Immunol., 172:6922-30, 2004.

23. K.H. Mills, Regulatory T cells: friend or foe in immunity to infection? Nat. Rev. Immunol., 4:841-55, 2004.

24. A.J. Macpherson and T. Uhr, Compartmentalization of the mucosal immune responses to commensal intestinal bacteria. Ann. NY Acad. Sci., 1029:36-43, 2004a.

25. A.J. Macpherson and T. Uhr, Induction of protective IgA by intestinal dendritic cells carrying commensal bacteria. Science, 303:1662-5, $2004 \mathrm{~b}$. 
26. M. Schultz, S. Watzl, T.A. Oelschlaeger, H.C. Rath, C. Göttl, N. Lehn, J. Schölmerich and H.J. Linde, Green fluorescent protein for detection of the probiotic microorganism Escherichia coli strain Nissle 1917 (EcN) in vivo. J. Microbiol. Methods, 61:389-98, 2005.

27. T. Matsuzaki, A. Takagi, H. Ikemura, T. Matsuguchi and T. Yokokura, Intestinal microflora: probiotics and autoimmunity. J. Nutr., 137:798S-802S, 2007.

28. P. Trevisi, S. De Filippi, L. Minieri, M. Mazzoni, M. Modesto, B. Biavati and P. Bosi, Effect of fructo-oligosaccharides and different doses of Bifidobacterium animalis in a weaning diet on bacterial translocation and Toll-like receptor gene expression in pigs. Nutrition, 24:1023-9, 2008.

29. C.A. Dogi, C.M. Galdeano and G. Perdigón, Gut immune stimulation by non pathogenic Gram(+) and Gram(-) bacteria. Comparison with a probiotic strain. Cytokine, 41:223-31, 2008.

30. M.G. Vizoso Pinto, M. Rodriguez Gómez, S. Seifert, B. Watzl, W.H. Holzapfel and C.M. Franz, Lactobacilli stimulate the innate immune response and modulate the TLR expression of HT29 intestinal epithelial cells in vitro. Int. J. Food Microbiol., 133:86-93, 2009.

31. A. Grabig, D. Paclik, C. Guzy, A. Dankof, D.C. Baumgart, J. Erckenbrecht, B. Raupach, U. Sonnenborn, J. Eckert, R.R. Schumann, B. Wiedenmann, A.U. Dignass and A. Sturm, Escherichia coli strain Nissle 1917 ameliorates experimental colitis via toll-like receptor 2- and toll-like receptor 4-dependent pathways. Infect. Immun., 74:4075-82, 2006.

32. C. Grangette, S. Nutten, E. Palumbo, S. Morath, C. Hermann , J. Dewulf, B. Pot, T. Hartung, P. Hols and A. Mercenier, Enhanced antiinflammatory capacity of a Lactobacillus plantarum mutant synthesizing modified teichoic acids. Proc. Natl. Acad. Sci. USA., 102:10321-6, 2005. 
33. S. Voltan, I. Castagliuolo, M. Elli, S. Longo, P. Brun, R. D'Incà, A. Porzionato, V. Macchi, G. Palù, G.C. Sturniolo, L. Morelli and D. Martines, Aggregating phenotype in Lactobacillus crispatus determines intestinal colonization and TLR2 and TLR4 modulation in murine colonic mucosa. Clin. Vaccine Immunol., 14:1138-48, 2007.

34. M. Miettinen, V. Veckman, S. Latvala, T. Sareneva, S. Matikainen and I. Julkunen, Live Lactobacillus rhamnosus and Streptococcus pyogenes differentially regulate Toll-like receptor (TLR) gene expression in human primary macrophages. J. Leukoc. Biol., 84:1092-100, 2008.

35. H. Jijon, J. Backer, H. Diaz, H. Yeung, D. Thiel, C. McKaigney, C. De Simone and K. Madsen, DNA from probiotic bacteria modulates murine and human epithelial and immune function. Gastroenterology, 126:1358-73, 2004.

36. J.B. Ewaschuk, J.L. Backer, T.A. Churchill, F. Obermeier, D.O. Krause and K.L. Madsen, Surface expression of Toll-like receptor 9 is upregulated on intestinal epithelial cells in response to pathogenic bacterial DNA. Infect. Immun., 75:2572-9, 2007.

37. J.M. Otte, E. Cario and D.K. Podolsky, Mechanisms of cross hyporesponsiveness to Tolllike receptor bacterial ligands in intestinal epithelial cells. Gastroenterology, 126:1054$70,2004$.

38. D. Kelly, J.I. Campbell, T.P. King, G. Grant, E.A. Jansson, A.G. Coutts, S. Pettersson and S. Conway, Commensal anaerobic gut bacteria attenuate inflammation by regulating nuclear-cytoplasmic shuttling of PPAR-gamma and RelA. Nat. Immunol., 5:104-12, 2004.

39. S. Voltan, D. Martines, M. Elli, P. Brun, S. Longo, A. Porzionato, V. Macchi, R. D'Incà, M. Scarpa, G. Palù, G.C. Sturniolo, L. Morelli and I. Castagliuolo, Lactobacillus crispatus M247-derived $\mathrm{H} 2 \mathrm{O} 2$ acts as a signal transducing molecule activating peroxisome proliferator activated receptor-gamma in the intestinal mucosa. Gastroenterology, 135:1216-27, 2008. 
40. P.A. Ruiz, M. Hoffmann, S. Szcesny, M. Blaut and D. Haller, Innate mechanisms for Bifidobacterium lactis to activate transient pro-inflammatory host responses in intestinal epithelial cells after the colonization of germ-free rats. Immunology, 115:441-50, 2005.

41. A.S. Neish, A.T. Gewirtz, H. Zeng, A.N. Young, M.E. Hobert, V. Karmali, A.S. Rao and J.L. Madara, Prokaryotic regulation of epithelial responses by inhibition of IkappaB-alpha ubiquitination. Science, 289:1560-3, 2000.

42. Y.P. Lin, C.H. Thibodeaux, J.A. Peña, G.D. Ferry and J. Versalovic, Probiotic Lactobacillus reuteri suppress proinflammatory cytokines via c-Jun. Inflamm. Bowel Dis., $14: 1068-83,2008$.

43. M. Lotz, D. Gütle, S. Walther, S. Ménard, C. Bogdan and M.W. Hornef, Postnatal acquisition of endotoxin tolerance in intestinal epithelial cells. J. Exp. Med., 203:973-84, 2006.

44. E. Heuvelin, C. Lebreton, C. Grangette, B. Pot, N. Cerf-Bensussan and M. Heyman, Mechanisms involved in alleviation of intestinal inflammation by Bifidobacterium breve soluble factors. PLoS One. 4(4):e5184, 2009.

45. W. Strober, A. Kitani, I. Fuss, N. Asano and T. Watanabe, The molecular basis of NOD2 susceptibility mutations in Crohn's disease. Mucosal Immunol., 1 Suppl 1:S5-9, 2008.

46. M. Cross, Microbes versus microbes: immune signals generated by probiotic lactobacilli and their role in protection against microbial pathogens. FEMS Inmunol. Medical. Microbiol., 34: 245-253, 2002.

47. I. Kato, K. Tanaka, and T. Yokokura, Lactic acid bacterium potently induces the production of interleukin-12 and interferon- $\gamma$ by mouse splenocytes. Int. $J$. Immunopharmacol., 21, 121-131, 1999.

48. M. Hoffmann, E. Rath, G. Hölzlwimmer, L. Quintanilla-Martinez, D. Loach, G. Tannock and D Haller, Lactobacillus reuteri 100-23 transiently activates intestinal epithelial cells 
of mice that have a complex microbiota during early stages of colonization. $J$ Nutr. ,138:1684-91, 2008.

49. D. Bosisio, N. Polentarutti, M. Sironi, S. Bernasconi, K. Miyake, G.R. Webb, M.U. Martin, A. Mantovani and M. Muzio, Stimulation of toll-like receptor 4 expression in human mononuclear phagocytes by interferon-gamma: a molecular basis for priming and synergism with bacterial lipopolysaccharide. Blood, 99:3427-31, 2002.

50. M. Medina, E. Izquierdo, S. Ennahar and Y. Sanz, Differential immunomodulatory properties of Bifidobacterium longum strains: relevance to probiotic selection and clinical applications. Clin. Exp. Immunol., 150:531-8, 2007.

51. O. Ménard, M.J. Batel, V. Gaboriau-Routhiau and A.J. Waligora-Dupriet, Gnotobiotic mouse immune response induced by Bifidobacterium sp. strains isolated from infants. Appl. Environ. Microbiol., 74:660-6, 2008.

52. T. Werner and D. Haller, Intestinal epithelial cell signalling and chronic inflammation: From the proteome to specific molecular mechanisms. Mutat. Res., 622:42-57, 2007.

53. A.C. Ouwehand, N. Bergsma, R. Parhiala, S. Latineen, M. Gueimonde, H. Finne-Soveri, T. Strandberg, K. Pitkälä and S. Salminen, Bifidobacterium microbiota and parameters of immune function in elderly subjects. FEMS Immunol. Med. Microbiol., 53:18-25, 2008.

54. K. Munakata, M. Yamamoto, N. Anjiki, M. Nishiyama, S. Imamura, S. Iizuka, K. Takashima, A. Ishige, K. Hioki, Y. Ohnishi and K. Watanabe, Importance of the interferon-alpha system in murine large intestine indicated by microarray analysis of comensal bacteria-induced immunological changes. BMC Genomics 9:192, 2008.

55. M.L. Forchielli and W.A. Walker, The role of gut-associated lymphoid tissues and mucosal defence. Br J Nutr., 93:S41-8, 2005.

56. D.C Baumgart and S.R. Carding, Inflammatory bowel disease: cause and immunobiology. Lancet, 369:1627-40, 2007. 
57. L. O'Mahony, L. O'Callaghan, J. McCarthy, D. Shilling, P. Scully, S. Sibartie, E. Kavanagh, W.O. Kirwan, H.P. Redmond, J.K. Collins and F. Sanan, Differential cytokine response from dendritic cells to commensal and pathogenic bacteria in different lymphoid compartments in humans. Am. J. Physiol. Gastrointest. Liver Physiol., 290:G839-45, 2006.

58. S.L. Young, M.A Simon, M.A. Baird, G.W. Tannock, R. Bibiloni, K. Spencely, J.M. Lane, P. Fitzharris, J. Crane, I. Town, E. Addo-Yobo, C.S. Murria and A. Woodcock, Bifidobacterial species differentially affect expression of cell surface markers and cytokines of dendritic cells harvested from cord blood. Clin Diagn Lab Immunol., 11:68690, 2004.

59. C. Hoarau, C. Lagaraine, L. Martin, F. Velge-Roussel and Y. Lebranchu, Supernatant of Bifidobacterium breve induces dendritic cell maturation, activation, and survival through a Toll-like receptor 2 pathway. J Allergy Clin Immunol., 117:696-702, 2006.

60. A.L. Hart, K. Lammers, P. Brigidi, B. Vitali, F. Rizzello, P. Gionchetti, M. Campieri, M.A. Kamm, S.C. Knight and A.J. Stagg. Modulation of human dendritic cell phenotype and function by probiotic bacteria. Gut, 53:1602-9, 2004.

61. T. Hrncir, R. Stepankova, H. Kozakova, T. Hudcovic and H. Tlaskalova-Hogenova, Gut microbiota and lipopolysaccharide content of the diet influence development of regulatory T cells: studies in germ-free mice. BMC Immunol. 9:65, 2008.

62. H.H. Smits, A. Engering, D. van der Kleij, E.C. de Jong, K. Schipper, T.M. van Capel, B.A. Zaat, M. Yazdanbakhsh, E.A. Wierenga, Y. van Kooyk and M.L. Kapsenberg, Selective probiotic bacteria induce IL-10-producing regulatory $\mathrm{T}$ cells in vitro by modulating dendritic cell function through dendritic cell-specific intercellular adhesion molecule 3-grabbing nonintegrin. J. Allergy Clin. Immunol., 115:1260-7, 2005. 
63. C. O'Mahony, P. Scully, D. O'Mahony, S. Murphy, F. O'Brien, A. Lyons, G. Sherlock , J. MacSharry, B. Kiely, F. Sanan and L. O'Mahony, Commensal-induced regulatory Tcells mediate protection against pathogen-stimulated NF-kappaB activation. PLoS Pathog. 4:e1000112, 2008.

64. B. Foligne, G. Zoumpopoulou, J. Dewulf, A. Ben Younes, F. Chareyre, J.C. Sirard, B. Pot and C.A. Grangette, A key role of dendritic cells in probiotic functionality. PLoS One, 2:e313, 2007.

65. S.K. Mazmanian, C.H. Liu, A.O. Tzianabos and D.L Kasper, An immunomodulatory molecule of symbiotic bacteria directs maturation of the host immune system. Cell, 122:107-18, 2005.

66. H. Majamaa, E. Isolauri, M. Saxelin and T. Vesikari, Lactic acid bacteria in the treatment of acute rotavirus gastroenteritis. J. Pediatr. Gastroenterol. Nutr., 20:333-8, 1995. 


\section{Captions for figures}

Figure 1. Signalling pathways triggered by the interaction of diverse bacterial components with the epithelium and GALT. Intestinal epithelial cells and antigen presenting cells (macrophages and dendritic cells [DCs]) express pattern recognition receptors, including the cell surface TLRs 2, 4 and 5 and the intracellular TLRs 3, 7 and 9. Lipoteichoic acids (LTA) from Gram-positive bacteria, LPS from Gram-negative bacteria, dsRNA from virus, flagellin from bacteria, RNA homologous (e.g. resiquimod R848) and ssRNA from viruses, and CpG oligonucleotides from bacterial and viral DNA are recognized respectively by TLR2, 4, 3, 5, 7 and 9. Upon-ligand binding, TLRs promote signal divergence via interactions with different adaptor proteins (MyD88, TIRAP [MAL], TRIF [TICAM1] and TRAM). MyD88 is used by all TLRs, except for TLR3; TIRAP I is used by TLR2 and 4, TRIF is used by TLR3 and 4 and TRAM is used only by TLR4. These interactions activate three major signalling pathways: the nuclear factor (NF)- $\mathrm{B}$, the mitogen-activated protein kinases (MAPKs) and IRFs. Common to all TLRs is activation of (NF)-kB and AP-1 through MAPKs, leading to the production of inflammatory cytokines and chemokines. Interferon regulatory factor (IRF) 3 and 7 are also activated by TLR3, 7 and 9, leading to the production of type I IFNs (IFN- $\alpha$ S and IFN- $\beta)$.

Figure 2. Differentiation of naïve $\mathrm{T}$ cells into $\mathrm{T}$ effector and regulatory $\mathrm{T}$ cells subpopulations by TLR-activated DCs. 